\title{
Reducing the Missing Wedge in TEM Tomography
}

\author{
A. Genc ${ }^{1}$, L. Kovarik ${ }^{2}$ L. Pullan ${ }^{1}$ and J. Ringnalda ${ }^{1}$ \\ 1. FEI Company, 5350 NE Dawson Creek Drive, Hillsboro, OR, USA \\ 2. Environmental Molecular Sciences Laboratory, Pacific Northwest National Laboratory, P.O. Box 999, \\ Richland, WA, USA
}

This work describes a new development for extending tilt capabilities in computed tilt tomography for transmission electron microscopy (TEM) in order to reduce the missing wedge artifacts in tomographic reconstructions of nanoparticle materials. TEM samples of nanoparticles were tilted beyond the conventional limit of $\pm 70^{\circ}$ tilt in an aberration corrected TEM. By improving the tilting capability of conventional $3 \mathrm{~mm}$ TEM samples within the pole piece gap of the microscope, more information can be extracted in 3D. Tomographic tilt series of gamma $(\gamma)$ alumina catalyst support were acquired at $5^{\circ}$ tilt increments to a maximum tilt angular range of $-85^{\circ} / 83^{\circ}$ (Figure 1b). Results of high visibility tilting has revealed a clear improvement in the 3D visualization of $\gamma$-alumina depicting crystallographic $\langle 111>$ faceting on the (110) surfaces of the nanoparticles. Crystallographic information parallel to the optic axis of the microscope has been improved by employing TEM tomography beyond the conventional $\pm 70^{\circ}$ tilt angular regime (Figure 1c). The tilt data thus obtained shows a reduction of up to $75 \%$ in the 'missing wedge' as compared to conventional single axis $\left( \pm 70^{\circ}\right)$ TEM tomography (Figure 1d). The resolution of the reconstructions in the image plane $\left(\mathrm{d}_{\mathrm{xy}}\right)$ is defined by the spatial resolution of the images, whereas parallel to optic axis $\left(\mathrm{d}_{\mathrm{z}}\right)$, the resolution is defined by the number of tilt increments in the tilt series [1]. Although a sufficient number of tilt increments may be used in the tilt series, valuable information parallel to optic axis is often missing in the 3D tomographic reconstructions because of the limits in the tilt capability of the microscope. Typically this is $\pm 70^{\circ}$ due to the objective lens pole piece gap of the microscope; the pole piece design (including the gap) defines the spherical aberration $\left(\mathrm{C}_{\mathrm{s}}\right)$ of the microscope [2]. By design, maximum angular tilt range vs resolution is a compromise, so for 'high tilt' systems it is mostly $\pm 70^{\circ}$ whereas narrower pole piece gaps for 'high resolution' provide smaller $\mathrm{C}_{\mathrm{s}}$ values to improve resolution in TEM. However this limits the available maximum tilt range to in some cases less than $30^{\circ}$ unless undesirable non-standard TEM samples are employed. Limited tilting of the sample results in missing of important tilt data parallel to the optic axis in the form of a missing wedge. Therefore 3D reconstructions obtained with limited tilts show streaking and distortion artifacts in the direction parallel to the optic axis. The resolution $\left(d_{z}\right)$ parallel to optic axis is defined by the elongation factor $\left(e_{y z}\right)$ as a function of maximum tilt angular range $\left(\alpha_{\max }\right)$ where $\alpha$ is in radians [1].

$$
\begin{aligned}
& d_{z}=d_{x y} e_{y z} \\
& e_{y z}=\sqrt{\frac{\alpha_{\max }+\sin \alpha_{\max } \cos \alpha_{\max }}{\alpha_{\max }-\sin \alpha_{\max } \cos \alpha_{\max }}}
\end{aligned}
$$

There have been many attempts to reduce missing wedge effects such as by machining pillar shaped samples from bulk materials, and in this manner achieve a full rotation of the sample in the narrow pole piece gap [3]. This approach showed constraints such as a limited field of view of the specimen, plus effects of sample preparation have to be considered. Many isolated nanostructures such as catalysts and nanomaterials (ex: nanotubes and rods) are difficult or impossible to be machined in a pillar shape by using a focused ion beam (FIB) or cannot be transferred easily for a full rotation in the microscope. More recently, mathematical models based on compressive sensing have been utilized as well to improve the quality of the 3D TEM tomographic reconstructions. However these results still suffer from the limited tilting of the TEM samples in the microscope. 


\section{References:}

[1] P.A. Midgley et al., Ultramicroscopy 96 (2003) 413-431

[2] M. Haider et al., Ultramicroscopy 75 (1998) 53-60

[3] N. Kawase et al., Ultramicroscopy 107 (2007) 8-15

(a)
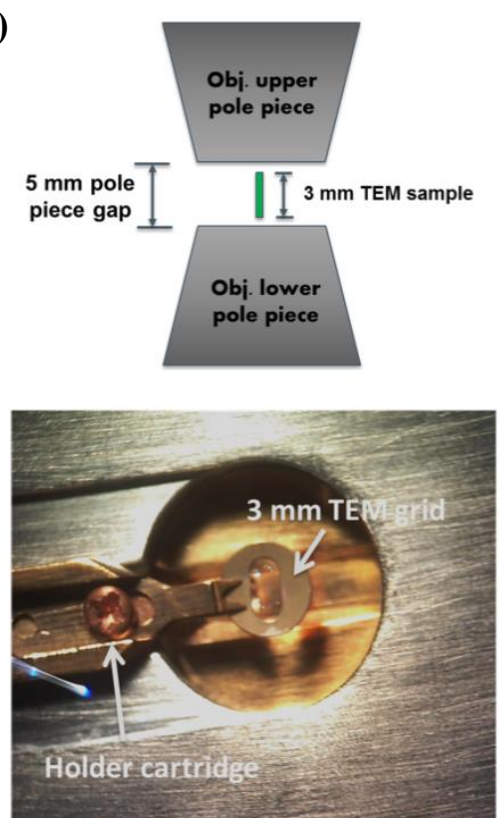

(d)

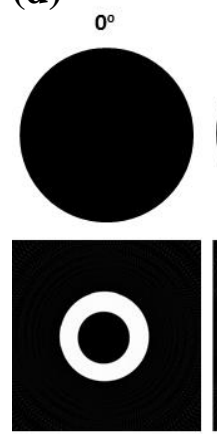

$\alpha_{\max }=90^{\circ}$

$e_{y z}=1$

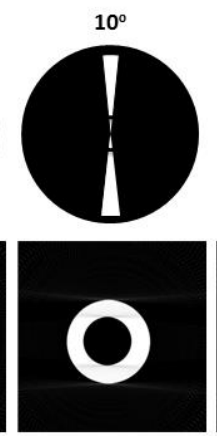

$\alpha_{\max }=\mathbf{8 5}^{\circ}$

$\mathrm{e}_{\mathrm{yz}}=1.1$

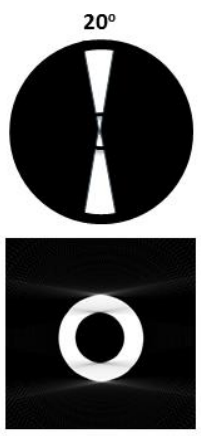

$\alpha_{\max }=80^{\circ}$

$\mathrm{e}_{\mathrm{yz}}=1.2$
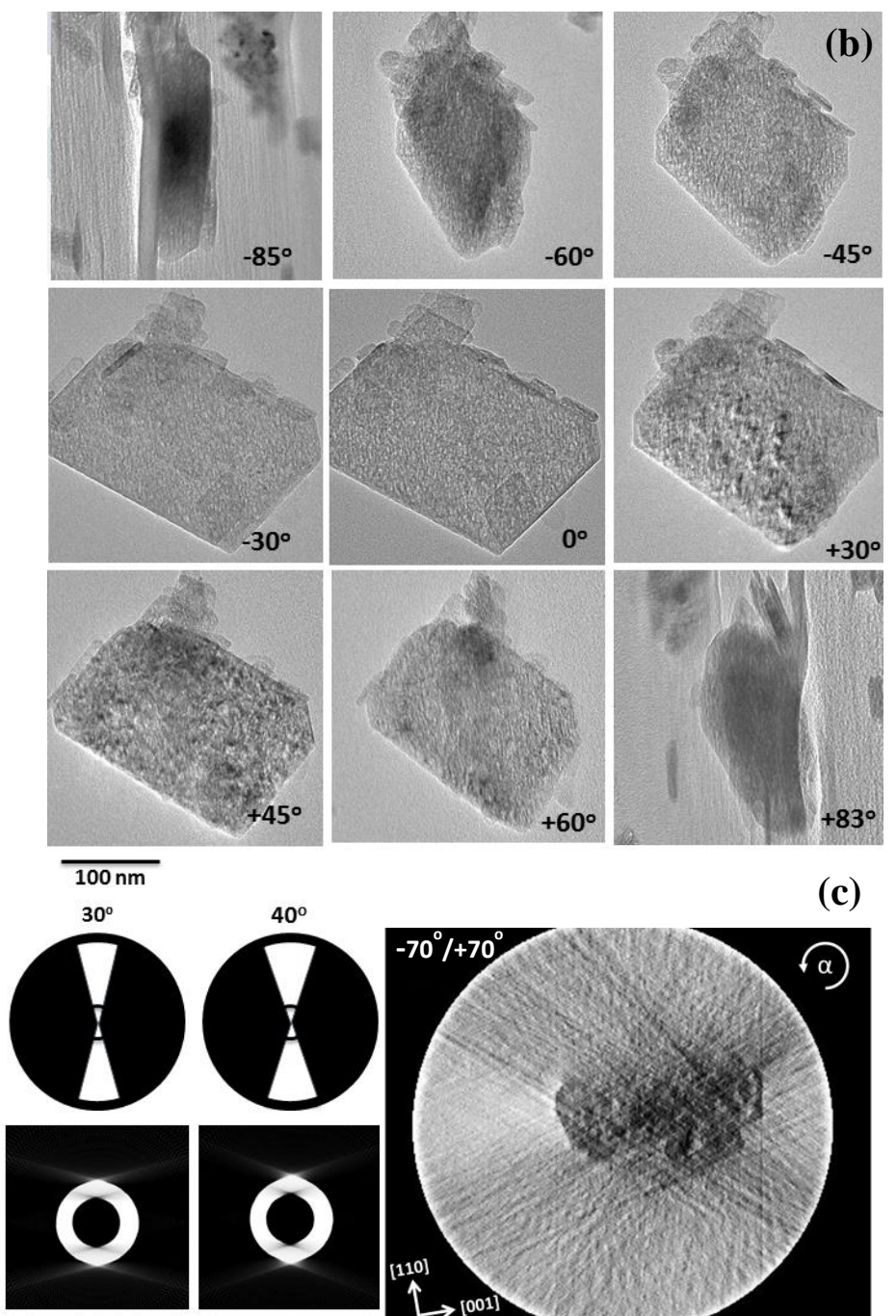

$\alpha_{\max }=75^{\circ}$

$\mathrm{e}_{\mathrm{yz}}=1.25$

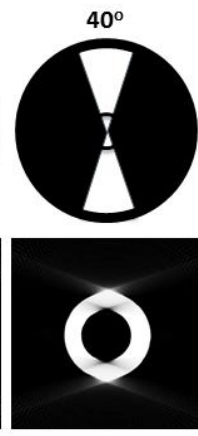

$\alpha_{\max }=70^{\circ}$

$e_{\mathrm{yz}}=1.3$

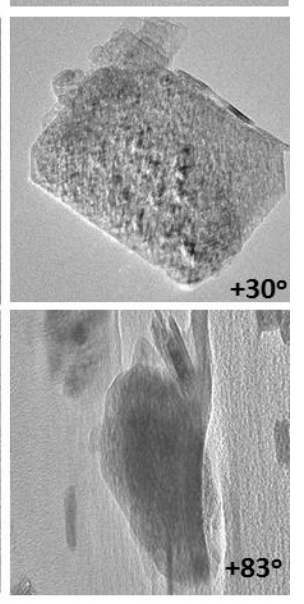

(c)

Figure 1. (a) $3 \mathrm{~mm}$ slot grid attached to high visibility tomography holder allowing high tilting (b) A sub-set of TEM tilt images acquired from a $\gamma$-alumina catalyst at $-85^{\circ} / 83^{\circ}$ (c) Orthogonal slices of the reconstructions (top) $\pm 70^{\circ}$ and $\mathrm{e}_{\mathrm{yz}}=1.3$ (bottom) $-85^{\circ} / 83^{\circ}$ and $\mathrm{e}_{\mathrm{yz}}=1.1$ at $5^{\circ}$ tilt increment (d) Simulated images of a ring shape with direct back projection algorithm 